\title{
ENSEIGNER LES ARTS PLASTIQUES EN ZEP : LES DESSOUS DU METIER
}

\author{
Laurence ESPINASSY $^{* *}$ et Frédéric SAUJAT ${ }^{*}$
}

Article paru dans « Recherche et Formation » $N^{\circ} 44,2003$, INRP

\begin{abstract}
Résumé
L'activité du professeur consiste moins en la réalisation d'une tâche d'enseignement qu'en sa re-conception, issue des arbitrages relatifs à la recherche d'une efficacité non seulement dans l'accomplissement de son travail, mais aussi dans l'accomplissement de soi. C'est ce point de vue que nous voudrions soutenir dans cet article, en nous appuyant sur l'analyse de l'activité de professeurs d'arts plastiques en ZEP. Nous montrerons que ces enseignants trouvent des solutions efficaces pour "prendre" et "faire" la classe, au double sens de milieu et de collectif de travail. Nous mettrons ensuite en évidence que l'organisation du travail des élèves passe par des gestes de métier puisant tout à la fois dans l'expérience individuelle et dans une mémoire trans-personnelle, constituée de façons de faire partagées, liées à la "gestion" spécifique de l'engagement psycho-corporel du professeur d'arts plastiques. Nous conclurons sur les perspectives qu'ouvre l'analyse du travail enseignant à la formation.
\end{abstract}

L'exercice professionnel en zones d'éducation prioritaires (ZEP) est une épreuve pour l'enseignant, au double sens de preuve (d'occasion de vérifier et d'affirmer son métier) et d'empêchement (de souffrance résultant d'une mise à mal du métier et d'une perte du pouvoir d'agir). Dès lors, la question de l'efficacité des pratiques enseignantes, qui apparaît aujourd'hui comme une préoccupation importante, tant pour la recherche en éducation que pour les politiques scolaires et la formation des maîtres, se pose en ZEP dans toute sa complexité.

En effet, l'évaluation de cette efficacité est le plus souvent envisagée à partir des effets produits sur les élèves en termes d'apprentissages, et non à partir du travail d'enseignement requis pour engendrer ces effets. Or, comme dans tout travail, l'activité de l'enseignant est orientée par la recherche d'une efficacité malgré tout (Clot, 1995 ; Amigues \& Saujat, 1999), sous la double impulsion du sens de cette activité pour le sujet, et de la nécessaire efficience des opérations permettant sa réalisation.

Autrement dit, on ne saurait selon nous appréhender l'efficacité du travail réalisé par un professeur, sans prendre en compte les arbitrages grâce auxquels il cherche à faire de ce

\footnotetext{
** Laurence Espinassy, UMR ADEF, Université de Provence, IUFM d'Aix-Marseille, INRP.

* Frédéric Saujat, UMR ADEF, Université de Provence, IUFM d'Aix-Marseille, INRP.
} 
travail un opérateur efficace de réalisation de soi (c'est-à-dire de sa compétence, de sa santé mentale et de son identité).

Nous voudrions montrer, dans cet article, que si ce deuxième "niveau" d'efficacité de l'activité enseignante est toujours problématique en ce qu'il renvoie aux complexes négociations de l'usage de soi (Schwartz, 2000), le premier "niveau" est loin d'avoir le caractère d'évidence que semblent lui donner nombre de travaux en définissant l'objet du travail professoral comme l'apprentissage des élèves.

En effet, comme le note Amigues (2004), "la guérison du patient est au médecin ce que l'apprentissage des élèves est au professeur : un but lointain et incertain", vers lequel tend son action. Cet apprentissage ne se fait ni dans l'instant, ni sans médiations. La tâche d'enseignement comporte "une forte composante dynamique et historique, car elle remplit moins un programme d'actions tout défini d'avance qu'elle rend possible, en quelque sorte, le futur en le réalisant" (Tardif \& Lessard, 1999, p.226).

De ce point de vue, si l'apprentissage constitue bien la cible de l'action du professeur, on ne saurait sous-estimer ce que ça lui demande du point de vue de son activité que de viser un tel “objet d'horizon”. Les compromis qu'il est contraint de passer nous renseignent sur le fait que le travail est toujours pour lui une expérience à travers laquelle il se mesure à lui-même et aux autres pour s'efforcer de réaliser ce qu'on lui demande. L'activité du professeur résulte donc, pour nous, des arbitrages et des compromis qui conduisent l'enseignant à composer avec luimême, avec ses conceptions et convictions professionnelles, comme avec ses élèves et ses collègues.

C'est ce que nous nous proposons d'examiner, en nous appuyant sur l'analyse de l'activité de deux professeurs d'Arts Plastiques en ZEP (notés A.P. par la suite) : nous soutiendrons que ce choix - l'enseignement d'une discipline aux caractéristiques particulières dans un contexte qui tend à pousser le métier dans ses retranchements -, constitue un révélateur de composantes du travail enseignant. Ces dernières, mises au jour de manière convergente par d'autres recherches (Amigues, 2004 ; Espinassy, 2003 ; Saujat, 2002), nous semblent revêtir un certain caractère générique. Nous essaierons d'en tirer quelques conséquences pour la formation.

\section{Comment analyser l'activité du professeur?}

L'ergonomie de l'activité enseignante telle que nous la concevons (Amigues, Faïta, \& Saujat, 2004 a) se fonde clairement sur l'approche historico-culturelle (Vygotski, 1934/1997). Cette 
dernière avance notamment que l'expérience et son développement ne sont pas immédiatement accessibles par l'observation directe, mais à partir de “ traces” qu'il faut reconstruire, ce qui nécessite le recours à des “ méthodes indirectes” (Vygotski, 1999; Clot, 2001).

En effet, l'expression de l'expérience professionnelle est tributaire d'une double épreuve : celle d'une mise en travail de la pensée des opérateurs à travers sa mise en mots, d'une part, et celle de la confrontation aux mots des autres avant leur réintégration dans leur potentiel discursif, d'autre part. C'est la raison pour laquelle nous proposons la création d'un espacetemps particulier où sont suspendues les différentes contraintes sociales pesant sur l'activité des sujets, de telle sorte que puissent émerger les non-dits initiaux, les sous-entendus et informulés inhibés par l'action. Ce moment consiste en la mise en place d'un dispositif d' “ autoconfrontation" (Faïta et Vieira, 2003 ; Amigues, Faïta, \& Saujat, 2004 b), faisant appel à l'enregistrement vidéo d'une séance de classe, auquel est confronté l'enseignant filmé (pour plus de détails sur ce dispositif voir Lataillade dans ce $\mathrm{N}^{\circ}$ ). Le chercheur suscite une controverse professionnelle dans laquelle chaque sujet peut " réaliser" ce qu'il fait en voyant faire l'autre et en étant questionné sur ses actes.

Les données qui servent de support à cet article sont issues de cette méthodologie: autoconfrontations simples ou croisées conduites avec deux professeurs d'A.P expérimentés, Éric exerçant en ZEP à Marseille, et Léa ayant débuté à Sartrouville. Les caractéristiques de ces contextes d'enseignement sont très proches : ZEP, zone sensible, prévention violence ...

\section{Du milieu de travail des élèves ...}

Les professeurs d'A.P. sont soumis à de fortes contraintes institutionnelles auxquelles s'ajoutent celles liées aux conditions matérielles et au contexte socio-économique des élèves de ZEP. Nous n'entrerons pas ici dans le détail de leur contenu, mais les programmes officiels sont assez flous concernant les directives de mise en œuvre: c'est donc l'enseignant qui choisit sa progression, les œuvres auxquelles il fera référence, qui invente ses dispositifs d'enseignement afin que chacun des élèves produise plastiquement à chaque heure de cours.

C'est bien là que réside la difficulté majeure : il est impératif que des réalisations soient visibles en fin de séance, car c'est à partir du débat qu'elles suscitent que se construit l'enseignement.

Considérant qu'un certifié d'A.P. effectue dix-huit heures de cours hebdomadaires, c'est un minimum de 450 élèves et donc autant de productions qui défilent dans la salle en une 
semaine. En imaginant les exigences de manutention, la "gestion" du temps et de l'espace que cela impose, on comprend mieux les préoccupations des professeurs d'A.P. concernant leur milieu de travail et la préservation de leur intégrité physique et psychique.

Cette heure unique d'enseignement hebdomadaire peut être d'autant moins "ratée", que le professeur ne peut compter, au maximum, que sur les trente-six séances de l'année scolaire, soit environ douze thématiques de travail différentes.

Le tour de force, a fortiori avec des élèves “difficiles”, consiste à ce qu'en une heure ils s'installent, comprennent la demande, s'en emparent, produisent, se mobilisent pour échanger autour des réalisations et enfin rangent; tout en respectant la parole des autres, celle du professeur comme celle des camarades de classe ...

Il s'agit donc pour l'enseignant d'instaurer un cadre tout à la fois solide et souple, pour offrir de bonnes conditions de travail et permettre des productions plastiques différentes.

Mais, au-delà de cette enveloppe matérielle, quand on observe sur l'enregistrement vidéo d'une séance de cours en ZEP, que des élèves de $6^{\text {ème }}$ en Janvier sont en mesure d'anticiper quand le professeur donne les consignes, c'est qu'ils ont déjà acquis de nombreuses règles de fonctionnement. Eric, le professeur filmé, a annoncé qu'il travaillait sur le long terme, il sait qu'il les aura jusqu'en $3^{\text {ème }}$ comme en témoignent ses paroles : “On est obligé de les structurer ..., en tout cas en $6^{\circ}$; mais ce n'est jamais gagné : c'est valable ensuite pour les $5^{\circ}$. Il n'y a qu'en $4^{\circ}-3^{\circ}$ qu'on peut espérer que $2 / 3$ des élèves sachent se servir d'un cahier de texte ...". Ce ne sont pas seulement des habitudes de travail qu'il leur donne, mais tout un style de façons de faire au prix d'un investissement initial coûteux, mais dont le bénéfice attendu est, nous dit-il, prometteur : “quitte à perdre du temps au début, tant pis, mais c’est un énorme gain de temps pour les années d'après ”.

De plus, quand les élèves se retrouvent en salle d'A.P, lieu d'étude et de vie dans lequel demeurent des traces de travaux anciens, qui "font mémoire" et servent de référence, ils savent pourquoi et comment se comporter là, par rapport aux autres et au travail que l'on y produit. En effet, nous émettons l'hypothèse que les efforts consentis à l'organisation du travail de la classe sont adressés autant aux élèves qu'à l'enseignant lui-même, et que les éléments du cadre mis en place sont autant de repères pour les élèves (surtout pour ceux qui en manquent), que pour le développement du travail du professeur. Il semblerait qu'en entrant dans une salle d'A.P, l'on sache qu ' “ en accédant à une communauté de ce genre, l'on n'est pas seulement entré dans un ensemble de conventions de praxis, mais dans une manière d'exercer l'intelligence" (Bruner 1996, p.192). Les conditions d'action en A.P. sont intimement liées aux conditions d'apprentissage. Les règles de fonctionnement au sein de la 
classe sont donc dictées par des nécessités d'action. Ces règles indispensables pour se partager les outils communs, (matériel, techniques, références, postures ...), ne sont pas seulement " disciplinaires" au sens du maintien de l'ordre; elles le sont aussi au sens de la discipline enseignée, car elles sont constitutives de l'activité de travail.

Dans cette perspective, les professeurs d'A.P disposent d'un outil professionnel appelé “incitation". Même si son usage est aujourd'hui largement diffusé dans les centres de formation, chaque enseignant le façonne lui-même pour en faire un instrument (Rabardel, 1995) qui lui permet tout à la fois d'enrôler chacun des élèves dans une tâche individuelle, de dynamiser l'accomplissement d'une œuvre commune et de faire acquérir des règles du métier d'élève.

\subsection{L'incitation}

Ce terme est peut-être une spécificité du jargon de l'enseignement des A.P. : il s'agit le plus souvent d'une petite phrase, accompagnée ou non d'un document, qui sert de déclencheur au cours. Il ne s'agit ni d'un “ énoncé " qui renvoie peut-être trop à l'idée d' “ exercice ”, ni d'un " sujet" qui se limiterait à une thématique à travailler. La " réussite" du cours dans toutes ses dimensions dépend de la qualité de l'incitation : en découlent la mise au travail rapide des élèves, leur motivation, leur respect du cadre, ..., autant d'éléments majeurs décrits plus haut. Pour mieux comprendre le fonctionnement de ce dispositif pédagogique, nous nous appuierons sur l'autoconfrontation de Léa, qui dit avoir tout appris du métier en ZEP où elle débuta, et en conserver depuis de nombreux gestes professionnels. Elle explique que c'est particulièrement vrai concernant le soin qu'elle porte à l'élaboration et à la rédaction de ses incitations.

On peut considérer la situation ci-après comme prototypique du fonctionnement de cet outil.

La séance de cours filmée, support des dialogues, débute ainsi : les élèves de $5^{\text {ème }}$, en entrant en classe, trouvent sur leur table une photocopie format A3 d'un plan de Marseille, quelques feuilles de papier ou de plastique aux degrés de transparence différents. Ils doivent travailler à deux. Une phrase est alors inscrite au tableau par Léa : “Il y a 2 vents à Marseille : celui qui apporte la pollution, celui qui la chasse". Un élève la lit à haute voix, le professeur fait remarquer que c'est bien une virgule qui articule la seconde partie de la phrase et aussitôt lance : “ Top chrono ! C'est parti !”. Les élèves se mettent au travail ...

Rappelons que l'un des objectifs incontournables en A.P. est que les élèves produisent puis débattent à partir de leurs travaux. Le “ cadrage " évoqué plus haut, l'est ici à nouveau quand 
Léa précise : “...du début jusqu'à la fin, tout repose sur leurs productions! Donc, ça n'aurait pas de sens si ce n'était pas dans le respect de chacun”. Pour les faire “ démarrer", elle propose à la fois des matériaux qui provoquent un effet de surprise et interrogent les élèves quant à leur éventuelle utilité, et cette petite phrase. Un va-et-vient permanent s'opère ensuite entre incitation et production, qui nécessite des conditions d'échanges, de dialogue, d'écoute.

Il s'agit pour les élèves de prendre à leur compte la situation conçue pour eux, par le professeur. Même si les choix de réponse s'avèrent multiples, ces différenciations ne peuvent exister qu'en rapport aux contraintes fixées par l'incitation. L'aspect différentiel des réalisations qu'elle permet est le support du dialogue entre professeur et élèves, mais aussi de ces derniers entre eux, du début à la fin du cours. C'est à cette occasion que se repèrent les choix techniques, les procédures engagées, les négociations pour répondre aux contraintes esthétiques: ici, par exemple, le choix des matériaux en fonction de leurs qualités expressives.

Les élèves savent qu'ils ont un rôle à jouer lié au fait qu'ils appartiennent à un " collectif de travail" au sens de Cru (1995), qui s'est constitué autour d'une œuvre commune à réaliser, (non pas au sens d'une réalisation plastique collective, mais d'une avancée des savoirs de la classe et des individus qui la composent). Cette œuvre commune mobilise les manières d'agir, de dire et de penser inscrites dans l'histoire didactique passée et à venir du groupe, et pour laquelle chacun est amené à participer. Dans ce cas, et considérant que tout se déroule dans l'unique heure de cours hebdomadaire (et normalement sans travail à la maison), aucun des élèves ne devrait régresser, ou se mettre à l'écart: chacun doit montrer qu'il avance non seulement comme les autres mais avec les autres.

L'incitation est donc génératrice d'un milieu de travail pour les élèves afin qu'ils étudient des questions, à l'aide de techniques qu'ils doivent s'approprier à travers l'usage qui en est fait en classe. On le voit, le milieu, la cohésion du groupe et le développement de l'activité sont indissociables. La classe n'est donc pas seulement un lieu d'interactions entre un enseignant, des élèves et un savoir. Ce " milieu-classe" assure les conditions propices au fonctionnement d'une communauté dont on partage "l'intelligence étendue" (Bruner 1996, p.192) et au sein de laquelle s'opère la construction conjointe du savoir enseigné et de l'identité de chacun. Mais nous l'avons évoqué, l'appartenance à un collectif sous-entend l'acceptation de certaines règles de fonctionnement. Envisageons maintenant le rôle majeur de l'incitation dans l'instauration de règles de travail, afin d'acquérir de la "compétence scolaire" (Bruner, 1996). 


\subsection{Des règles de métier}

Les "règles de métier" sont davantage des termes de psychologues du travail que de didacticiens. Nous allons néanmoins nous efforcer de montrer que l'incitation, au-delà du strict apprentissage disciplinaire en A.P., joue un rôle décisif dans l'organisation du travail des élèves.

Il était prévu que les élèves travaillent par groupe de deux. Comme attirés par des aimants, les deux plus agités de la classe se retrouvent à la même table. Lors de son autoconfrontation, le chercheur demande à Léa si cette situation ne risque pas de poser problème. C'est alors qu'elle rappelle "l'histoire de deux vents" et que "travailler à deux ça a du sens"; elle précise qu'elle a fait le choix du groupe à cause “des objectifs d'A.P. ” qui sont ici en jeu, relevant des "savoir-faire notionnels" inscrits dans les prescriptions officielles, mais auxquels elle a associé “ des objectifs de savoir-être liés implicitement à l'incitation ”. Léa précise qu'il s'agit que les deux élèves perturbateurs et récidivistes " deviennent ces 2 vents" et que même s'ils ne "passent pas par les objectifs d'A.P." ils auront " traversé" l'éducation à la citoyenneté “ dans des objectifs comme ça”. Elle est contente de constater à l'écran que " finalement, paradoxalement, je ne peux pas dire qu'ils sont brillants, mais agissants au moins, concernés".

Même si quelques rappels à l'ordre sont nécessaires, force est de constater que ces deux élèves travaillent, négocient entre eux la gestion et le choix des matériaux. Visiblement intéressés, ils sont partie prenante dans la logique du cours, et si leur participation à la réalisation collective est minime, c'est déjà un succès aux yeux de Léa.

A propos de l'incitation, elle signale qu'elle prend le risque que cette dernière " tombe à plat", mais espère surtout qu'elle puisse "faire moteur ... ". Léa fait référence à plusieurs reprises au cours de l'autoconfrontation à ce phénomène d'entraînement que l'on attribuerait à une courroie ou une chaîne de transmission, sauf qu'il s'agit de faire avancer ici non pas un véhicule mais toute une classe vers de nouveaux savoirs.

Cette idée d'interaction permanente entre les individus et les événements de la classe se retrouve à plusieurs niveaux ; ainsi elle déclare à propos de la discipline à faire respecter : “..., je ne vais pas m'arrêter pour prendre un carnet, ou ce genre de chose. J'essaie plutôt de les mettre dans le mouvement ...". C'est bien sur cette notion essentielle de mouvement qu'elle insiste, persuadée nous semble-t-il, que l'avancée du savoir ne peut se faire sans la participation des élèves. Plus loin, ce n'est plus la métaphore mécanique qui est employée, mais c'est au domaine de la physique des particules que Léa nous renvoie en nous invitant à 
"vraiment chercher dans l'incitation le noyau qui va dynamiser..." et qui, par une réaction en chaîne, "va faire sens après au niveau de l'évaluation!".

Il s'agit donc, pour Léa, de faire vivre un collectif de travail, dont le fonctionnement suppose de la part des élèves un cheminement individuel, de la connaissance des règles à leur intériorisation. Selon Cru (1995), “ la règle, en psychopathologie du travail n'a pas valeur en regard de sa fonction d'unification, d'identification ou de défense du groupe. Elle vaut par le type de médiation qu'elle entretient entre l'individu et le groupe : la règle permet à chacun de cultiver sa singularité sans nuire à la réalisation du travail commun ” (p.61).

Nous retrouvons-là les ambitions de l'enseignement des A.P. cherchant à autoriser chacun à produire de façon singulière tout en participant à la construction collective des savoirs de la classe.

Nous faisons l'hypothèse que c'est l'imbrication des gestes professionnels permettant de "prendre" la classe avec ceux qui permettent de la "faire" qui rend possible le développement de ce processus dans la classe de Léa. Mais c'est aussi, au-delà des élèves, par ces gestes que Léa s'inscrit dans des manières communes de faire et de penser le métier ...

\section{III ... Au métier du professeur d'A.P.}

Il semble que pour les professeurs d'A.P c'est plus particulièrement la reconnaissance de l'efficacité de ces gestes "imbriqués" qui permet l'identification entre eux comme appartenant au même collectif (Espinassy, 2003). Les enseignants de cette discipline sont la plupart du temps seuls à l'exercer dans leur établissement; pourtant malgré cet éparpillement géographique et des formations peu homogènes, ils se retrouvent autour de façons communes de faire et de penser leur métier.

C'est donc qu'il existe pour les professeurs d'A.P, comme pour les collectifs de travail d'élèves, des règles qui “ contraignent les marginaux par rapport au métier, [en même temps qu'elles] libèrent ceux qui les acceptent de toute hésitation, de toute inquiétude paralysante" (Cru, 1995, p. 55). Elles sont des balises, des repères qui assurent une fonction protectrice et structurante.

Les efforts consentis à l'organisation du travail de la classe sont adressés autant aux élèves qu'à l'enseignant lui-même, dans la mesure où le cadre institué vise à rendre possible le travail des élèves tout autant que celui du professeur. 
L'analyse de l'activité de Léa nous met tout particulièrement sur la voie de l'existence de cette activité psycho-corporelle (Scheller \& Clot, 2001) par laquelle le professeur s'efforce de mobiliser son métier pour interpréter, utiliser ou transformer son milieu de travail.

En effet, à la faveur d'un arrêt sur image, on voit Léa passer quelques instants, penchée, à discuter avec une tablée d'élèves. Léa explique alors qu'elle conçoit ses cours comme un vaste mouvement, une circulation d'énergies allant du professeur vers les élèves et inversement. Elle-même incarne tout à fait cette dynamique: elle ne s'est, par exemple, jamais assise durant les cinquante-cinq minutes de la séance filmée. Tout se passe pour elle comme s'il fallait mettre et maintenir un système en ordre de marche. Cette mise en action évoque tout autant un parcours mental intangible, que sa manifestation dans la réalité matérielle de la classe : les déplacements ou changements d'attitude des élèves aussi bien que du professeur, en sont les indicateurs. Ces positionnements autant physiques qu'intellectuels, Léa les nomme souvent "postures".

Elle commente ainsi la séquence où l'on peut la voir accroupie, les yeux au niveau de la table des élèves avec lesquels elle discute : “Donc, la parole et le regard, ça fait deux va et vient, qui demandent peut-être une posture plus agissante! Supposons qu'il y ait des réalisations sur la table : si je me mets debout, ils vont me regarder comme ça, puis leur travail, et puis moi leur travail, puis eux... Alors que là, la posture est vraiment liée au jeu de regards et à ce qu'il doit se passer : c'est-à-dire, au niveau du regard '. D'après Léa, le corps et les mouvements du professeur prennent naturellement place dans la logique du cours et il lui semblerait même anormal qu'on ne se baisse pas pour se mettre au niveau des travaux des élèves. C'est pour elle une façon de décentrer l'attention des élèves puis de la recentrer sur leur travail : une technique pédagogique élaborée, une sorte de dialogue didactique muet. Elle va plus loin encore, expliquant au chercheur qu'à ses yeux en A.P. les enseignants n'ont pas tout à fait la même attitude que leurs collègues : “On a des comportements différents par rapport à eux, on a une motricité complètement différente : on circule, etc...". Notons le caractère générique de ses propos (usage du “on"), traduisant sa conviction de la nécessité de ces déplacements. Nécessité professionnelle que Léa aborde à plusieurs reprises : “En allant, comme ça prendre des informations, des amorces de projets au tout début du cours, et en reconduisant l'opération, ça va me permettre de fédérer quelques groupes autour de mes objectifs ... Alors que ce petit travail, d'aller de l'un à l'autre, en fait c'est pour tisser ! ...”. C'est donc un travail de mobilisation des troupes, de régulation du travail et d'harmonisation de l'avancée commune de la réflexion qui est accompli ici. Outre cette importance majeure pour la dynamique de ses cours, sa mobilité est aussi directement opératoire. Par exemple, 
elle annonce qu'elle n'a pas besoin de faire l'appel pour savoir s'il y a un absent car par “ $l e$ fait d'être nomade dans la classe, on calibre vite qui il manque". Cette façon de lier sans cesse la gestion de la classe et celle des contenus est une préoccupation permanente : son "nomadisme" lui permet à tous moments de connaître l'état du groupe et de l'avancement du travail.

En réinscrivant la logique des savoirs enseignés dans celle de la "mise au travail des élèves", l'ergonomie de l'activité enseignante entend insister sur le caractère collectif et négocié des actions à réaliser, sur leur dimension temporelle, et sur la part d'engagement subjectif qu'elles réclament de l'enseignant. Dans le cas des professeurs d'A.P. avec lesquels nous avons travaillé, il semble bien que cet engagement passe par des modalités spécifiques, qui sont reconnues comme des manières de faire partagées : en A.P. il ne s'agirait pas d'une belle machine bien huilée qui tourne sans à-coups, mais pour Léa “ du mouvement en permanence!".

Ce serait donc là à la fois l'une des richesses et des fragilités de cette discipline, où comme le dit Léa, il faut "gérer" sans cesse cette énergie et ses éventuels débordements, exigeant une vigilance et une mobilité particulières de la part du professeur.

Ces modalités particulières de "gestion", qui requièrent aussi du professeur "quelque chose comme un "se" gérer" (Schwartz, 2000, p. 425), sont configurées dans ce qu'on proposera de qualifier de "genre professeurs d'arts plastiques" (Espinassy, 2003). Nous entendons par là, par homologie avec la notion de "genres de discours" chez Bakhtine (1984), moins une norme professionnelle achevée qu'un système ouvert de façons de faire partagées et liées à des préoccupations communes. Ce système fonctionne comme une mémoire trans-personnelle qui alimente les recréations stylistiques de chacun lesquelles, après validation par le métier, contribuent en retour à entretenir la vitalité de cette mémoire.

Pour parler comme Canguilhem, les "infidélités" du milieu de travail que constitue la classe pour le professeur, réclament de ce dernier qu'il y mette du sien pour retoucher des ressources que le genre met à sa disposition et qui ne répondent plus aux exigences de son action : c'est cette mobilisation subjective que l'existence d'un cadre a précisément pour fonction de rendre possible. "L'énergie" déployée pour que ce cadre " tienne", surtout en ZEP, pour coûteuse et éprouvante qu'elle soit, trouve donc sa source dans les efforts consentis par l'enseignant pour maintenir et développer sa santé au travail, entendue là encore avec Canguilhem (1984) comme l'affirmation d'une normativité par laquelle tout sujet cherche à la fois à se maintenir en vie et à accroître son pouvoir d'agir. Autrement dit, la fonction protectrice et structurante du cadre ne vaudrait pas que pour les élèves, il semblerait qu'elle soit recherchée par le 
professeur comme une condition du développement de sa propre activité, incarnée dans des “dramatiques” d'efficacité (Schwartz, 2000), au cœur desquelles il s'efforce de puiser auprès des autres et en lui-même les ressources nécessaires pour faire entrer en résonance accomplissement de son travail et accomplissement de soi.

Le souci de se préserver n'est donc pas seulement une question d'agrément personnel (même si le fait de regagner son domicile sans fatigue excessive n'est pas négligeable), mais bien une affaire d'efficacité professionnelle : il faut pouvoir enchaîner classe après classe et tenir jusqu'en fin de journée. "Savoir durer" s'inscrit dans une liste de préoccupations professionnelles qui, bien que non inscrites officiellement au titre de “compétence ", n'en sont pas moins essentielles dans l'exercice du métier au quotidien.

C'est ce que met en avant Eric, lors de l'autoconfrontation: "J'en suis à ma lle année d'enseignement et il y a des choses que je ne faisais pas au début et sur lesquelles je suis revenu de manière systématique pour mon confort dans ma vie de tous les jours, et pour la qualité du cours. [...] Un cours d'A.P. complètement lâche, dans ce genre d'endroits, avec des gamins aussi jeunes et aussi peu structurés, c'est à mon avis... suicidaire, c'est faire du n'importe quoi! S'il y a une faille dans un système mis en place, les élèves s'y engouffrent la tête la première, et c'est le prof qui se fait manger à la petite cuillère. Moi j'ai envie de renter chez moi, d'accueillir les classes d'après, d'avoir des journées correctes!”.

Le fait d'imbriquer des gestes professionnels permettant de " prendre ", “ faire " et " tenir" sa classe, offre en retour à l'enseignant une économie d'énergie non négligeable qui, s'il conserve ses classes d'une année sur l'autre, comme c'est le cas des professeurs d'A.P., peut être réinvestie à long terme.

\section{CONCLUSION}

L'enseignement des A.P., de surcroît en ZEP, produit avec d'autres disciplines comme l'éducation physique et sportive par exemple, un effet de loupe sur une caractéristique générique du travail du professeur : il s'agit en effet pour ce dernier de faire faire pour faire apprendre par les élèves. On sait que ce "passage" du faire à l'apprendre ne va pas de soi : il peut être difficile à négocier pour un certain nombre d'élèves et générer des "malentendus" qui affectent le sens même du travail scolaire (Bautier \& Rochex, 1997). Mais c'est ici que le détour par l'analyse du travail du professeur peut conduire à regarder autrement la façon dont la production de ces "malentendus" opère dans l'activité enseignante. 
Il ressort de nos analyses, que la contrainte de faire faire pour faire apprendre place le professeur dans la situation paradoxale d'un prescripteur de tâches, d'un organisateur du travail qui devrait simultanément, qu'il le veuille ou non et en temps réel, réorganiser cette tâche en fonction de l'activité des élèves qui la réalisent; et ce, tout en favorisant le travail collectif de ces élèves sur l'organisation même de cette tâche. On peut alors comprendre le sentiment de fatigue qui résulte souvent d'un exercice professionnel soumis à une telle “charge psychique", tout particulièrement dans ces zones de turbulence (Davisse, 1996) où se trouve propulsée aujourd'hui une partie des enseignants .

En effet, si la cible de l'action professorale est bien, comme nous l'avons dit, l'apprentissage des élèves, l'atteinte de cette cible n'est jamais directe ni immédiate : elle passe par les efforts des enseignants pour avoir une "classe qui tourne”, c'est-à-dire qui puisse faire s'épauler cohésion du groupe et cohérence des acquisitions. Ces efforts sont guidés par une sorte d'intelligence des situations professionnelles qui se développe au fil de l'expérience et se matérialise dans des gestes professionnels, dont l'efficacité dynamique est tournée à la fois vers les élèves et vers l'enseignant lui-même, comme sujet actif qui arbitre entre ce qu'on lui demande et ce que ça lui demande (Noulin, 1995).

Ce que révèle l'analyse de l'activité professorale, c'est l'épaisseur des “couches de signification" (Billiard, 2001) liées à l'enchevêtrement des composantes sociales, collectives, techniques, corporelles, émotionnelles et subjectives en fonction desquelles se construit le sens de son travail pour le professeur (Saujat, 2002). Il incombe alors à ce dernier la tâche d'organiser cette pluralité de cadres de significations pour ne pas être condamné à l'inefficacité ou à l'absurde (Malrieu, 1983, p. 39).

Il y a sans doute là matière à réfléchir aux liens possibles entre analyse du travail enseignant et formation. Cette dernière est souvent conçue à partir d'une double analyse : celle des effets attendus du travail du professeur sur les élèves d'une part, et celle des savoirs (disciplinaires, didactiques, relevant des sciences humaines et sociales ...) supposés nécessaires au professeur pour produire de tels effets. Ce qui est ainsi "négligé", ce sont les situations concrètes de travail dans lesquelles se réalise (à tous les sens du terme) l'objet de l'activité du professeur, les milieux et les collectifs de travail au sein desquels se forment et se transforment les instruments de cette activité, et enfin les effets en retour de cette activité sur le professeur.

De ce point de vue, l'analyse du travail enseignant peut constituer un instrument psychologique rendant possible une autre "réalisation" (en conservant de nouveau la polysémie du terme) de l'activité du professeur. L'efficacité malgré tout (Amigues \& Saujat, 1999 ; Clot, 1995), au principe des choix opérés par l'enseignant dans son travail “ordinaire”, 
peut trouver dans le milieu "extra-ordinaire" de l'analyse du travail une opportunité de développement. Les échanges dialogiques que ce nouveau milieu suscite et nourrit conduisent en effet le professeur à considérer avec d'autres yeux cette efficacité malgré tout: les négociations et les arbitrages sur lesquels elle repose, résultant des conflits et des dilemmes qui "travaillent" l'activité, se révèlent comme des choix non définitifs, qui deviennent discutables précisément parce qu'ils sont discutés.

La voie est alors ouverte aux controverses sur le métier: plus précisément la discipline, d'outil pour mettre les élèves au travail dans l'activité “ordinaire" des professeurs, peut redevenir un objet de pensée collectif. Cette dialectique outil/objet ramène au premier plan les questions du sens de l'activité : si l'analyse du travail constitue une zone potentielle de développement par rapport à ce questionnement, il incombe à la formation d'organiser les apprentissages qui permettront aux intéressés de se réapproprier leur discipline afin d'en faire à nouveau un outil plus efficace, pour leurs élèves comme pour eux-mêmes. C'est-à-dire un outil qui développe à la fois l'efficacité dans le travail réalisé auprès des élèves, et l'efficacité de l'usage de soi dans la réalisation de ce travail.

\section{REFERENCES BIBLIOGRAPHIQUES}

Amigues, R., Faïta,. D \& Saujat, F. (2004 a). Travail enseignant et apprentissages scolaires. In E. Gentaz et Ph. Dessus (Eds.). Comprendre les apprentissages : Psychologie cognitive et éducation. Paris : Dunod (à paraître).

Amigues, R., Faïta, D. \& Saujat, F. (2004 b). "L'autoconfrontation croisée " : une méthode pour analyser l'activité enseignante et susciter le développement de l'expérience professionnelle. Bulletin de Psychologie (à paraître).

Amigues, R. \& Saujat, F. (1999). La formation initiale des professeurs vue par les intéressés : une approche ergonomique. Actes du Troisième Congrès International d'Actualité de la Recherche en Education, AECSE, Bordeaux.

Bakhtine, M. (1984). Esthétique de la création verbale. Paris : Gallimard.

Bautier, E. \& Rochex, J.Y. (1997). Apprendre : des malentendus qui font la différence. In J.P. Terrail (Ed.). La scolarisation de la France. Critique de l'état des lieux. Paris : La Dispute.

Billiard, I. (2001). Santé mentale et travail. L'émergence de la psychopathologie du travail. Paris : La Dispute.

Bruner, J. (1996). L'éducation, entrée dans la culture. Paris : Retz.

Canguilhem, G. (1984). Le normal et le pathologique. Paris : PUF.

Clot, Y (1995). Le travail sans l'homme? Paris : La Decouverte.

Clot, Y. (2001). Méthodologie en clinique de l'activité. L'exemple du sosie. In M. S. Delefosse \& G. Rouan (Eds.). Les méthodes qualitatives en psychologie. Paris : Dunod.

Cru, D. (1995). Règles de métier, langue de métier: dimension symbolique au travail et démarche participative de prévention. Mémoire EPHE, Paris. 
Davisse, A. (1996). L'EPS des ZEP... et d'ailleurs. In A. Davisse (Ed.). Lettres à nos remplaçants. Paris : Revue EPS.

Espinassy, L. (2003). Analyse de l'activité des professeurs d'arts plastique au collège. In Amigues, R., Faïta, D. \& Kherroubi, M. (Eds). Métier enseignant, organisation du travail et analyse de l'activité. Skholê, $n^{\circ}$ spécial (à paraître).

Faïta, D. \& Vieira, M. (2003). Réflexions méthodologiques sur l'autoconfrontation croisée. In Amigues, R., Faïta, D. \& Kherroubi, M. (Eds). Métier enseignant, organisation du travail et analyse de l'activité. Skholê, $n^{\circ}$ spécial (à paraître).

Malrieu, P. (1983). Genèse réciproque de l'idéologie et de la personnalisation. Psychologie et Education, 1-2, 35-42.

Noulin, M. (1995). L'analyse de l'activité : connaissance, compréhension, rencontre... Performances Humaines et Techniques, hors série septembre, 7-10.

Rabardel, P. (1995). Les hommes et les technologies, approche cognitive des instruments contemporains. Paris : Armand Colin.

Saujat, F. (2002). Ergonomie de l'activité enseignante et développement de l'expérience professionnelle : une approche clinique du travail du professeur. Thèse de Doctorat.Université de Provence, Aix-en-Provence, 13/12.

Schwartz, Y. (2000). Le paradigme ergologique ou un métier de philosophe. Toulouse : Octarès.

Scheller, L. \& Clot, Y. (2001). Comment la pensée se réalise dans l'activité de travail. In F. Hubault (Ed.). Comprendre que travailler c'est penser, un enjeu industriel de l'intervention ergonomique. Toulouse: Octarès.

Tardif, M. \& Lessard, C. (1999). Le travail enseignant au quotidien. Expérience, interactions humaines et dilemmes professionnels. De Boeck Université.

Vygotski, L. (1934/1997). Pensée et langage. Paris : Editions Sociales.

Vygotski, L. (1999). La signification historique de la crise en psychologie. Lausanne \& Paris: Delachaux et Niestlé. 